\title{
On the scaling of the solar incident flux
}

\author{
C. A. Varotsos ${ }^{1}$, S. Lovejoy ${ }^{2}$, N. V. Sarlis ${ }^{3}$, C. G. Tzanis $^{1}$, and M. N. Efstathiou ${ }^{1}$ \\ ${ }^{1}$ Climate Research Group, Division of Environmental Physics and Meteorology, Faculty of Physics, \\ University of Athens, University Campus Bldg. Phys. V, Athens, 157 84, Greece \\ ${ }^{2}$ Physics Department, McGill University, 3600 University St. Montreal, Que. H3A 2T8, Canada \\ ${ }^{3}$ Department of Solid State Physics, Faculty of Physics, School of Science, National and Kapodistrian \\ University of Athens, Panepistimiopolis Zografos, 15784 Athens, Greece
}

Correspondence to: C. A. Varotsos (covar@phys.uoa.gr)

Received: 20 February 2015 - Published in Atmos. Chem. Phys. Discuss.: 15 April 2015

Revised: 18 June 2015 - Accepted: 22 June 2015 - Published: 07 July 2015

\begin{abstract}
It was recently found that spectral solar incident flux (SIF) as a function of the ultraviolet wavelengths exhibits $1 / f$-type power-law correlations. In this study, an attempt was made to explore the residues of the SIF with respect to the Planck law over a wider range of wavelengths, from 115.5 to $629.5 \mathrm{~nm}$. Using spectral, Haar and Detrended Fluctuation analyses, we show that over the range from 10 $20 \mathrm{~nm}$ to the maximum lag $(\approx 500 \mathrm{~nm})$, the SIF residues have a scaling regime with fluctuation exponent $H \approx 0.37$ but with high intermittency $\left(C_{1} \approx 0.16\right.$, multifractal index $\alpha \approx 1.7$ ) and spectral exponent $\approx 1.46$. Over the shorter wavelengths range we found on the contrary low intermittency $\left(C_{1} \approx 0\right)$ with spectral exponent $\approx 1$ and $H \approx 0$.
\end{abstract}

\section{Introduction}

As is well known, electromagnetic radiation is continuously emitted by every physical body. This radiation is described by Planck's law near thermodynamic equilibrium at a definite temperature. There is a positive correlation between the temperature of an emitting body and the Planck radiation at every wavelength. As the temperature of an emitting surface increases, the maximum wavelength of the emitted radiation increases too. Smith and Gottlieb (1974) re-examined the subject of photon solar flux and its variations vs. wavelength and showed that variations in the extreme ultraviolet (UV) spectrum and in the X-ray of solar flux may reach high orders of magnitude causing significant changes in the Earth's ionosphere, especially during major solar flares (Kondratyev et al., 1995; Kondratyev and Varotsos, 1996; Ziemke et al.,
2000; Varotsos et al., 2001; Melnikova, 2009; Tzanis et al., 2009; Xue et al., 2011; Cracknell et al., 2014).

Solanki and Unruh (1998) proposed simple models of the total solar irradiance variations vs. wavelength showing that variations on solar flux are mainly caused by magnetic fields at the solar surface. Solar observations may be reproduced by a model of three parameters: the quiet Sun, a facular component and the temperature stratification of sunspots.

Tobiska et al. (2000) developed a forecasting solar irradiance model, called SOLAR2000, covering the spectral range of $1-1000000 \mathrm{~nm}$. Using this tool, the authors attempted to describe solar variation vs. wavelength and through time from X-ray through infrared wavelengths, in order to predict the solar radiation component of the space environment.

Very recently, Varotsos et al. (2013a, b) suggested the existence of strong persistent long-range correlations in spectral space of the solar flux fluctuations for UV wavelengths in the range $278-400 \mathrm{~nm}$. More precisely, by applying the detrending fluctuation analysis (DFA) to the initial SIF vs. UV wavelengths data set, power-law correlations of the type $1 / f$ were found pointing to a scaling feature in the UV spectral domain.

However, Varotsos et al. (2013a) tried to formulate the above-shown finding, i.e., that the solar spectral irradiance obeys $1 / f$ power-law as a function of UV wavelength, using the well-known Planck's law: $I(\lambda, T)=\frac{2 h c^{2}}{\lambda^{5}\left(e^{\frac{h c}{\lambda k T}}-1\right)}$ which, in the limit of small wavelengths tends to the Wien approximation: $I(\lambda, T)=\frac{2 h c^{2}}{\lambda^{5}} e^{-\frac{h c}{\lambda k T}}$, where $I(\lambda, T)$ is the amount of energy emitted at a wavelength $\lambda$ per unit surface area per unit time per unit solid angle per unit wavelength, $T$ is the 
temperature of the black body, $h$ is Planck's constant, $c$ is the speed of light, and $k$ is Boltzmann's constant. By applying the DFA method on the various values of $I(\lambda, T)$, Varotsos et al. (2013a) showed that the calculated $I(\lambda, T)$ values do not obey the $1 / f$-type scaling vs. $\Delta \lambda$ (i.e., for fluctuations in $I(\lambda, T)$ over a range $\Delta \lambda$ in wavelength). Thus, the latter may reflect a scaling in its fluctuations which might be related to the complex physical processes taking place at the solar atmosphere (e.g. see Avrett and Loeser, 2008, and references therein).

In the present study, focusing on these fluctuations, we examine whether the $1 / f$ scaling feature is apparent in a wider spectrum of SIF including both UV and visible spectrum, namely for wavelengths (WL) between 115.5 and $629.5 \mathrm{~nm}$.

\section{Data and analysis}

As mentioned above, the solar incident flux data for WL ranging from 115.5 to $629.5 \mathrm{~nm}$ with a step of $1 \mathrm{~nm}$ were analyzed. The spectrophotometric data of spectral extraterrestrial solar flux have been taken from the book by Makarova et al. (1991) (see also Makarova et al., 1994; Melnikova and Vasilyev, 2005). Figure 1a depicts SIF values for the wavelength range of $115.5-629.5 \mathrm{~nm}$. The principal feature shown in this figure is the existence of apparent nonstationarities vs. WL into the solar spectral distribution and the strong upward trend up to about $450 \mathrm{~nm}$. The detrending of this data set was accomplished (Fig. 1a) by applying the Planck formula $B_{1}\left(\frac{b_{1}}{\lambda}\right)^{5} /\left[\exp \left(\frac{b_{1}}{\lambda}\right)-1\right]$ with $b_{1}=2486.4 \mathrm{~nm}$ based on the Sun's effective temperature $(T$ sun $=5778 \mathrm{~K})$ reported by NASA (http://nssdc. gsfc.nasa.gov/planetary/factsheet/sunfact.html) and the derived parameter was found to be $B_{1}=85.8 \pm 0.7(0.82 \%)$ $\mathrm{mWm}^{-2} \mathrm{~nm}^{-1}$. Hereafter, we focus on these detrended SIF data (i.e. the residuals with respect to the Planck function) which are shown with the blue line in Fig. 1a; these are the deviations from a pure black body spectrum. To estimate the scaling exponents, we first applied the well-known DFA method (Peng et al., 1994; Weber and Talkner, 2001; Varotsos, 2005; Varotsos et al., 2008, 2009, 2011, 2012; Sarlis et al., 2010; Skordas et al., 2010; Efstathiou and Varotsos, 2010, 2013; Efstathiou et al., 2011; Chattopadhyay and Chattopadhyay, 2014).

Furthermore, we calculated the power spectrum for the detrended SIF data set using the Fast Fourier Transform (FFT) algorithm as well as the maximum entropy method (MEM) (Hegger et al., 1999).

A brief description of DFA- $n$ tool may be given as follows:

1. Consider the SIF data set $x(i)$ of length $N$ which is integrated over WL. Therefore, the integrated data set, $y(i)$, consists of the following points:
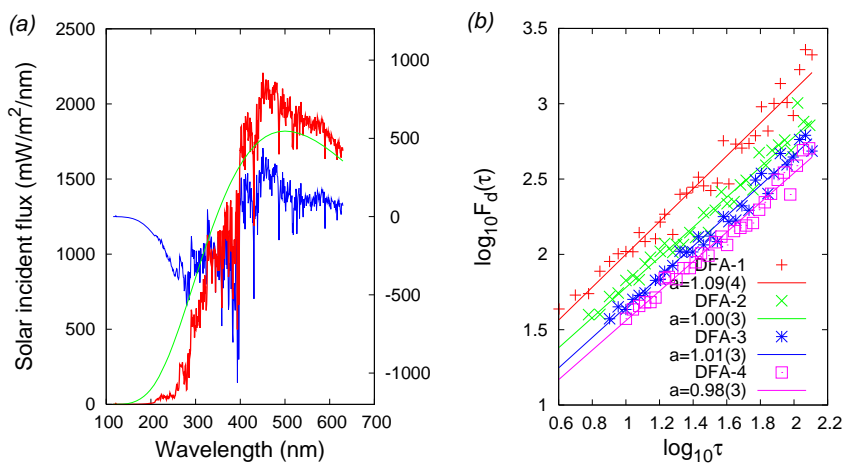

Figure 1. (a) SIF values (red, left scale) on the top of the atmosphere vs. WL from 115.5 to $629.5 \mathrm{~nm}$ together with the fitting employed (green, left scale). The detrended SIF data (blue, right scale) are also shown. (b) Log-log plot of the root mean square fluctuation function $F_{d}(\tau)$ of the detrended SIF vs. the WL segment size $\tau$, for the wavelengths between 115.5 and $629.5 \mathrm{~nm}$. The $a$ values for DFA-1, DFA-2, DFA-3, DFA-4 are $1.09( \pm 0.04), 1.00( \pm 0.03), 1.01$ $( \pm 0.03), 0.98( \pm 0.03)$, respectively.

$$
\begin{aligned}
& y(1)=x(1), \quad y(2)=x(1)+x(2) \ldots, \\
& y(i)=\sum_{k=1}^{i} x(k)
\end{aligned}
$$

2. We split the integrated data set into non-overlapping boxes of equal length, $\tau$. In each box, a best polynomial local trend (of order $n$ ) is fitted in order to detrend the integrated profile (by subtracting the locally fitted trend).

3. The root mean square fluctuations $F_{d}(\tau)$ of this integrated and detrended profile is calculated over all scales (box sizes). For each interval, the detrended fluctuation $F_{k}$ within the $k$ th box (Kantelhardt et al., 2002) is defined by:

$$
\begin{aligned}
& F_{k}^{2}(\tau)=\frac{1}{\tau} \sum_{i=k \tau+1}^{(k+1) \tau}[y(i)-z(i)]^{2}, \\
& k=0,1,2, \ldots, \quad\left(\frac{N}{\tau}-1\right),
\end{aligned}
$$

where $z(i)$ is the corresponding polynomial least-square fit to the $\tau$ data contained and (Peng et al., 1994):

$$
F_{d}^{2}=\frac{1}{(N / \tau)} \sum_{k=0}^{(N / \tau)-1} F_{k}^{2}(\tau) .
$$

In case the signals involve scaling, a power-law behavior for the root mean square fluctuation function $F_{d}(\tau)$ is observed: 
$F_{d}(\tau) \sim \tau^{a}$,

where $a$ is the scaling exponent, a self-affinity parameter that represents the long-range power-law correlation (Ausloos and Ivanova, 2001).

The above is fine for the nonintermittent, quasi-Gaussian case, but in the general (multifractal) case - which is of interest here - it is more convenient to define the mean fluctuation of the running sum $F_{d}^{\prime}$ using the mean $F_{d}^{\prime}=$ $\frac{1}{(N / \tau)} \sum_{k=0}^{(N / \tau)-1} F_{k}(\tau)$ in Eq. (3) rather than $F_{d}$ which is the RMS fluctuation of the running sum. Defining the mean fluctuation exponent $a^{\prime}$ by: $F_{d}^{\prime} \sim \tau^{a^{\prime}}$, we find that in the quasi Gaussian case, $a^{\prime}=a=$ the usual Hurst exponent $H$, although if we want the $H$ of the process (rather than its sum), we must subtract unity: $a=a^{\prime}=1+H$. In the general multifractal case, we can define the fluctuations of the process by $F_{d}^{\prime} / \tau$ and we can quantify the statistics via the $q$ th order structure function $S_{q}(\tau)=\left\langle\left(F_{d}^{\prime} / \tau\right)^{q}\right\rangle \sim \tau^{\xi(q)}$. We see that $a=1+\xi(2) / 2$ whereas $a^{\prime}=1+\xi(1)$. Finally, we can decompose $\xi(q)$ into linear and convex parts: $\xi(q)=$ $q H-K(q)$ where $K(q)$ characterizes the intermittent, multifractal contribution. With this, we obtain $a=1+H-\xi(2) / 2$, $a^{\prime}=1+H$; the two are equal when $K(q)=0$. In this special case where the process is quasi-Gaussian - unlike the general multifractal case - a single exponent is sufficient to characterize the scaling of the process. If the process is exactly Gaussian, when $0<a<1,(-1<H<0)$, the process is a fractional Gaussian noise (fGn) and when $1<a<2$ $(0<H<1)$, it is a fractional Brownian motion ( $\mathrm{fBm})$.

For uncorrelated quasi Gaussian data, the scaling exponent is $a=1 / 2,(H=-1 / 2)$. Scaling generally implies the existence of long range statistical dependencies. However in the special Gaussian case, when $a=1 / 2$, then one has a Gaussian white noise and there are no long range dependencies. However, the fact that $a=1 / 2$ can only be used to infer an absence of long range dependencies if it can be shown that the process is indeed quasi-Gaussian (in practice it has to be shown that it is not significantly intermittent, i.e. multifractal) in a certain range of $\tau$ values. If $0<a<0.5$ (and if the data set is nonintermittent), power-law anticorrelations are present (antipersistence). When $0.5<a \leq 1.0$ (and if again the data set is nonintermittent), then persistent long-range power-law correlations prevail (the case $a=1$ corresponds to the so-called $1 / f$ noise) (Weber and Talkner, 2001).

Finally, the scaling properties of SIF-WL data set were also studied using Haar fluctuation analysis (Lovejoy and Schertzer, 2012a, b). In the DFA method above, fluctuations are defined by the standard deviation of the residues of the polynomial regressions of the integrated process (the $F_{k}$; to obtain the fluctuation of the series rather than its sum, we must divide by $\left.\tau: F_{k}(\tau) / \tau\right)$. In the more general framework of wavelets, they are defined by convolutions with respect to (almost) arbitrarily shaped mother wavelets. While both DFA and wavelet based fluctuations give essentially the same accuracy when used for estimating scaling exponents (such as $H$, see e.g., Lovejoy and Schertzer, 2012a), if the wavelet is appropriately chosen, then the interpretation of the fluctuations becomes very simple so that the analysis can be used much more generally (i.e. when there is more than a single scaling regime, or even if there is no scaling at all). The difficulty in interpreting the DFA fluctuations is the reason why in published plots, units are typically not even provided for the fluctuation axes. In contrast, for the series $x(t)$ the simple (and indeed first wavelet) Haar fluctuations $\Delta x(\Delta t)$ are defined simply as the difference between the averages of a series over the first and second halves of an interval $\tau=\Delta t: \Delta x(\Delta t)=$ $2\left[\sum_{t+\Delta t / 2<t^{\prime}<t+\Delta t} x\left(t^{\prime}\right)-\sum_{t<t^{\prime}<t+\Delta t / 2} x\left(t^{\prime}\right)\right] / \Delta t(\Delta t$ is the time scale, time lag, it is the same as the $\tau$ in the DFA method). They have the property that in regions where $-1<$ $H<0$, they can be interpreted simply as resolution $\Delta t$ anomalies. Whereas in regions where $0<H<1$, they can be interpreted as differences: $\Delta x(\Delta t)=[x(t+\Delta t)-x(t)]$. Once the Haar fluctuations have been determined one can characterize them statistically by taking averages of various powers $q$; the generalized $q$ th order structure function $S_{q}(\Delta t)=\left\langle\Delta x(\Delta t)^{q}\right\rangle$, where the symbol $\langle$.$\rangle stands for en-$ semble averaging. In a scaling regime, $S_{q}(\Delta t) \approx \Delta t^{\xi(q)}$, where the exponent $\xi(q)=q H-K(q)$ and $K(q)$ is in general non-linear and convex and characterizes the intermittency (satisfying $K(1)=0$ ). In the previous DFA discussion, the process was assumed to be quasi-Gaussian, this implies the assumption $K(q)=0$ (the DFA fluctuations are of course valid without this restriction). For universal multifractals (e.g. Schertzer and Lovejoy, 1987),

$K(q)=C_{1} \frac{\left(q^{\alpha}-q\right)}{(\alpha-1)}$,

where $C_{1}(\geq 0)$ indicates the scaling intermittency and $\alpha(0 \leq \alpha \leq 2)-$ not to be confused with the DFA exponent - is the multifractality index (the Lévy index of the generator). Note that in the DFA literature, sometimes the exponent $h(q)=1+\xi(q) / q$ is introduced, the motivation being that in quasi Gaussian process, $K(q)=0$ so that $h(q)=$ constant (independent of $q$ ). However, when $C_{1} \neq 0$ this leads to $h(q) \rightarrow \infty$ as $q \rightarrow 0$ for all $\alpha \leq 1$. Since $\alpha=0$ is the monofractal limit, we obtain $h(0)=\infty$ which is neither intuitive nor convenient.

\section{Discussion and results}

Varotsos et al. (2013a) studying the high-resolution observations of SIF reaching the ground and the top of the atmosphere, suggested that SIF vs. UV WL exhibits $1 / f$-type power-law correlations. This result was derived by applying the DFA method on the SIF data set obtained from the Villard St. Pancrace station of the Lille University of Sciences and 

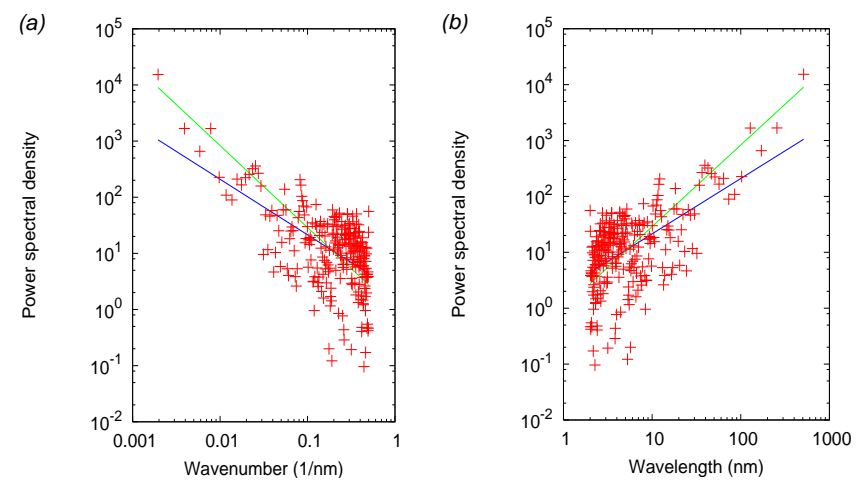

Figure 2. Power spectral density of the detrended SIF data set together with the least squares fit with power-law exponent $\beta=0.99$ ( \pm 0.08 ) (blue line) and the $\beta=1.46$ as determined with the Haar analysis (green line) vs. (a) the wavenumber, and (b) the wavelength.

Technology and was based on the slope (i.e., $a=1.02 \pm 0.02$, hence $H=0.02 \pm 0.02$ ) of the $\log -\log$ plot of the root mean square fluctuation function of SIF vs. the WL segment size $\tau \equiv \Delta t=\Delta \lambda$.

In the present study, the scaling dynamics of a wider spectrum of SIF vs. WL data set was studied, for wavelengths between 115.5 and $629.5 \mathrm{~nm}$. Firstly, DFA- $n$ seemed to take care of the trends revealing a DFA-exponent close to unity (after DFA-1), as shown in Fig. 1b.

In the following we plotted the power spectral density (using FFT) of the detrended SIF data set. The derived power spectral density showed that the power-law fitting gives an exponent $\beta=0.99( \pm 0.08)$ (see also below and Fig. 2). We can see that the slope inferred from the Haar analysis (below, $\beta=1.46$ ) also fits quite well, this is discussed below. In terms of the structure function exponent, we have the relation $\beta=1+\xi$ (2) which follows from the Wiener-Khintchin theorem (the spectrum is the Fourier transform of the autocorrelation function, which is a second order statistic, hence the $q=2$ ). On the other hand, the DFA-1 exponent was 1.09 ( \pm 0.04 ), while by applying the DFA- $n$ with $n>1$ on the detrended SIF data, the derived exponents ranged from 0.98 to 1.01 .

Next, to summarize our results we analyzed the detrended SIF-WL data set by using Haar analysis (Lovejoy and Schertzer, 2012a, b) using the software available at http://www.physics.mcgill.ca/ gang/software/doc/ haarpack.zip. According to Haar analysis, as also mentioned in Sect. 2, the variation of the SIF fluctuations vs. wavelength $\tau$ can be defined using the generalized $q$ th order structure function $S_{q}(\tau)=\left\langle\Delta \operatorname{SIF}(\tau)^{q}\right\rangle$, for which it holds that in a scaling regime $S_{q}(\tau) \approx \tau^{\xi(q)}$, where the exponent $\xi(q)=q H-K(q)$ and $K(q)$ illustrates the scaling intermittency (satisfying $K(1)=0$ and $\xi(1)=H$ ). Figure 3 b shows that $H=0.37$ and that the intermittency of SIF data set is very high $\left(C_{1}=0.16\right.$, also, $\left.\alpha \approx 1.7\right)$, hence the RMS exponent
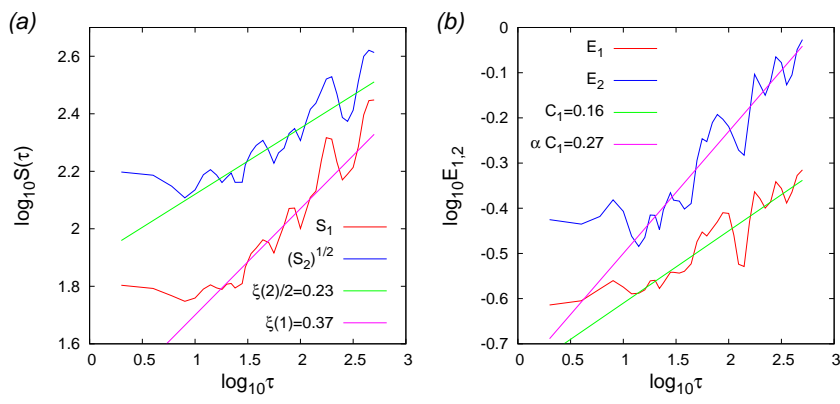

Figure 3. (a) Haar analysis on the detrended SIF data set for moments $q=1$ (red) and RMS (blue). $\xi(1)=\xi(2) \approx 0$ at scales below $10-20 \mathrm{~nm}$, whereas for larger scales up to $500 \mathrm{~nm}$ we have slopes $\xi(2) / 2=0.23$ (green) and $\xi(1)=H=0.37$ (magenta). (b) Red: $\log -\log$ plot of $E_{1}=S_{1} /\left(S_{1+\delta q} / S_{1-\delta q}\right)^{\frac{1}{2 \delta q}}$ vs. $\tau$ whose slope is $K^{\prime}(1)\left(=C_{1}\right)$, blue: $\log -\log$ plot of $E_{2}=\left(S_{1+2 \delta q} S_{1-2 \delta q} / S_{1}^{2}\right)^{-\frac{1}{4 \delta q^{2}}}$ vs. $\tau$ whose slope is $K^{\prime \prime}(1)\left(=\alpha C_{1}\right)$ for $\delta q=0.1$ (see Lovejoy and Schertzer, 2013, Ch. 10 for these estimators). The first yields an estimate $C_{1} \approx 0.16$ indicating high intermittency, the ratio yields an estimate $\alpha=1.7$. For smaller scales (up to $10-20 \mathrm{~nm}$ ), the corresponding slopes are close to 0 indicating Gaussianity. With these parameters and Eq. (5) (i.e., the universal multifractal equation for $K(q)$ in Sect. 2), we find $\xi(2) / 2=H-K(2) / 2 \approx 0.37-0.14=$ 0.23 in agreement with the direct estimate in Panel (a).

$=\xi(2) / 2=0.23$ is quite different from the $q=1$ exponent $(H)$ and the data are far from Gaussian. In the classical quasiGaussian case, $K(q)=0$ so that $\xi(q)$ is linear. More generally, if the field is intermittent - for example if it is the result of a multifractal process - then the exponent $K(q)$ is generally non-linear and convex and characterizes the intermittency, as already mentioned. The physical significance of $H$ is thus that it determines the rate at which mean fluctuations grow $(H>0)$ or decrease $(H<0)$ with scale $\tau$. According to Fig. 3a and b, the exponent $\xi(2)$ of the structure function equals to zero (at scales below $20 \mathrm{~nm}$ ), a fact which means that the power spectrum exponent $\beta=1+\xi(2)$ equals to 1 $(1 / f$ structure). On the other hand, at larger scales, the exponents $\xi(2)$ and $\beta$ seem to equal to 0.46 and 1.46 , respectively.

To clarify this aspect, we revisited the results of DFA1 (see Fig. 4a) and calculated the power spectrum for the detrended SIF-WL data set, using the MEM (see Fig. 4b). In Fig. 4a, we plot the root mean square fluctuation function $F_{d}(\tau)$ of DFA-1 together with the corresponding leastsquares fits for $\tau$ up to $15 \mathrm{~nm}$ (blue) - leading to $a=0.91$ $( \pm 0.08)$ - and above $20 \mathrm{~nm}$ (green) with $a=1.20( \pm 0.09)$. We observe a crossover approximately at $\tau=23 \mathrm{~nm}$, leading to $\beta$ values (cf., $\beta=2 a-1$, Talkner and Weber, 2000) above and below this crossover scale which are comparable to those found in the previous paragraph. In order to complement this finding, we plotted in Fig. 4b the MEM power spectral density vs. WL together with the aforementioned algebraic behaviors for WL above and below $20 \mathrm{~nm}$. Interestingly, the results show that the $\beta=1.46$ exponent better describes the 

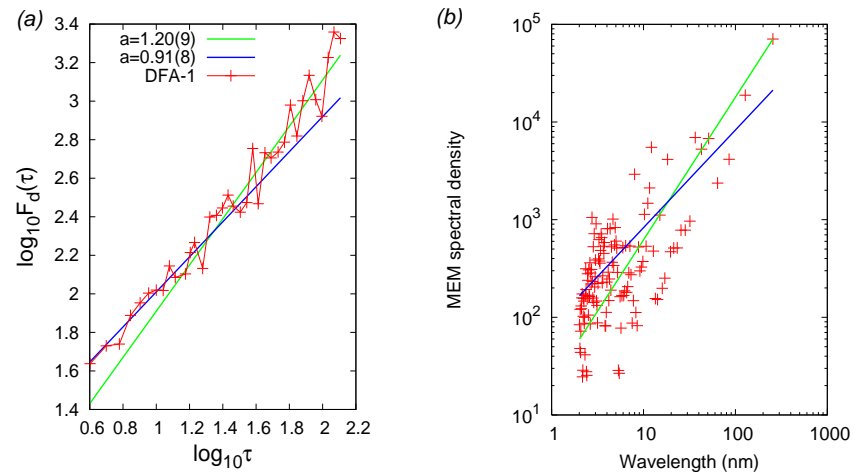

Figure 4. (a) Log-log plot of the root mean square fluctuation function $F_{d}(\tau)$ of the detrended SIF vs. the WL segment size $\tau$ for DFA-1 together with corresponding least-squares fits for $\tau \leq 15 \mathrm{~nm}$ (blue) and $\tau>20 \mathrm{~nm}$ (green). (b) Power spectrum using the MEM for the detrended SIF data set with the two power-law behaviors fits $\beta=1$ (blue) - from a least squares fit up to $20 \mathrm{~nm}$ - and $\beta=1.46$ (green) for the $1 / f$ and $1 / f^{1.46}$ structure, respectively, vs. the wavelength.

long WL body of the spectrum while the $\beta=1$ the short WL part of it.

We observe that the DFA method gives results similar to those of the Haar analysis, but obscures the break that is clearly seen in the Haar analysis (Fig. 3). Finally, we have to recall that the $1 / f$ scaling dynamics observed in SIF concerns not Planck's law but the fluctuations about the law.

\section{Conclusions}

The main conclusions of the present survey were:

1. DFA- $n$ applied on the detrended SIF data set revealed DFA-exponents close to unity. In other words, the SIF fluctuations around the Planck's law obey the $1 / f$ scaling dynamics.

2. Power spectral density for the detrended SIF data set showed that the power-law fitting gives $\beta=0.99$ $( \pm 0.08)$ while DFA-1 exponent was $a=1.09( \pm 0.04)$ and DFA- $n$ exponents ranged from 0.98 to 1.01 .

3. To better understand our results we analyzed the detrended SIF-WL data set by using Haar analysis. As it was derived, the intermittency of SIF data set was very high and the data were far from Gaussian. Specifically, the parameter characterizing the intermittency near the mean $C_{1}$ was $\approx 0.16$ and $\alpha \approx 1.7$. In comparison, in atmospheric turbulence, the wind field has $C_{1} \approx 0.07$ (also with $\alpha \approx 1.7$ ). At scales below $20 \mathrm{~nm}$, the power spectrum exponent $\beta$ was almost $1(1 / f$ structure $)$, while at larger scales, the exponents $\xi(2)$ and $\beta$ are equal to 0.46 and 1.46 , respectively. This prompted us to revisit DFA-1 and search for such a crossover. Indeed a crossover at $20 \mathrm{~nm}$ can be observed leading to compatible $\beta$ exponents. The DFA method analyzed the running sum rather than the process directly; this made the scale break (crossover) very difficult to discern.

4. The results of the power spectral density for the detrended SIF-WL data set (using the MEM) vs. frequency are compatible with the aforementioned two $\beta$ exponents.

Acknowledgements. This study was partly funded by Greek General Secretariat for Research and Technology (GSRT) through the project $12 \mathrm{CHN} 350$.

Edited by: A. Hofzumahaus

\section{References}

Ausloos, M. and Ivanova, K.: Power-law correlations in the southern-oscillation-index fluctuations characterizing El Niño, Phys. Rev. E, 63, 047201, doi:10.1103/PhysRevE.63.047201, 2001.

Avrett, E. H. and Loeser, R.: Models of the solar chromosphere and transition region from SUMER and HRTS observations: formation of the extreme-ultraviolet spectrum of hydrogen, carbon and oxygen, Astrophys. J. Suppl. S., 175, 229-276, 2008.

Chattopadhyay, G. and Chattopadhyay, S.: Study on statistical aspects of monthly sunspot number time series and its long-range correlation through detrended fluctuation analysis, Indian J. Phys., 88, 1135-1140, 2014.

Cracknell, A. P., Varotsos, C. A., and Timofeyev, Y. M.: Remote sensing of atmospheric radiation and dynamics, Int. J. Remote Sens., 35, 5563-5565, 2014.

Efstathiou, M. N. and Varotsos, C. A.: On the altitude dependence of the temperature scaling behaviour at the global troposphere, Int. J. Remote Sens., 31, 343-349, 2010.

Efstathiou, M. N. and Varotsos, C. A.: On the 11 year solar cycle signature in global total ozone dynamics, Meteorol. Appl., 20, 72-79, 2013.

Efstathiou, M. N., Tzanis, C., Cracknell, A. P., and Varotsos, C. A.: New features of land and sea surface temperature anomalies, Int. J. Remote Sens., 32, 3231-3238, 2011.

Hegger, R., Kantz, H., and Schreiber, T.: Practical implementation of nonlinear time series methods: the TISEAN package, Chaos, 9, 413-435, 1999.

Kantelhardt, J. W., Zschiegner, S. A., Koscielny-Bunde, E., Havlin, S., Bunde, A., and Stanley, H. E.: Multifractal detrended fluctuation analysis of nonstationary time series, Physica A, 316, 87-114, 2002.

Kondratyev, K. Y. and Varotsos, C. A.: Global total ozone dynamics-Impact on surface solar ultraviolet radiation variability and ecosystems, Environ. Sci. Pollut. R., 3, 205-209, 1996.

Kondratyev, K. Y., Pokrovsky, O. M., and Varotsos, C. A.: Atmospheric ozone trends and other factors of surface ultraviolet radiation variability, Environ. Conserv., 22, 259-261, 1995. 
Lovejoy, S. and Schertzer, D.: Haar wavelets, fluctuations and structure functions: convenient choices for geophysics, Nonlin. Processes Geophys., 19, 513-527, doi:10.5194/npg-19-513-2012, 2012a.

Lovejoy, S. and Schertzer, D.: Stochastic and scaling climate sensitivities: solar, volcanic and orbital forcings, Geophys. Res. Lett., 39, L11702, doi:10.1029/2012GL051871, 2012b.

Lovejoy, S. and Schertzer, D.: The Weather and Climate: Emergent Laws and Multifractal Cascades, Cambridge Univ. Press, Cambridge, UK, 496 pp., 2013.

Makarova, E. A., Kharitonov, A. V., and Kazachevskaya, T. V.: Solar Radiation Flux, Nauka Publ., Moscow, Russia, 400 pp., 1991 (in Russian).

Makarova, E. A., Kazachevskaya, T. V., and Kharitonov, A. V.: On the variability of some characteristics of solar radiative flux, Sol. Phys., 152, 195-200, 1994.

Melnikova, I.: Comparative assessment of the impact of molecular scattering and total ozone content on the characteristics of UV radiation in the atmosphere, Int. J. Remote Sens., 30, 6141-6150, 2009.

Melnikova, I. N. and Vasilyev, A. V.: Short-Wave Solar Radiation in the Earth's Atmosphere: Calculation, Observation, Interpretation, Springer/GmbH \& Co. KG, Berlin/Heidelberg, Germany, 2005.

Peng, C. K., Buldyrev, S. V., Havlin, S., Simons, M., Stanley, H. E., and Goldberger, A. L.: Mosaic organization of DNA nucleotides, Phys. Rev. E, 49, 1685-1689, 1994.

Sarlis, N. V., Skordas, E. S., and Varotsos, P. A.: Nonextensivity and natural time: the case of seismicity, Phys. Rev. E, 82, 021110, doi:10.1103/PhysRevE.82.021110, 2010.

Schertzer, D. and Lovejoy, S.: Physical Modeling and Analysis of Rain and Clouds by Anisotropic Scaling Multiplicative Processes, J. Geophys. Res. 92, 9692-9714, 1987.

Skordas, E. S., Sarlis, N. V., and Varotsos, P. A.: Effect of significant data loss on identifying electric signals that precede rupture by detrended fluctuation analysis in natural time, Chaos, 20, 033111, doi:10.1063/1.3479402, 2010.

Smith, E. V. P. and Gottlieb, D. M.: Solar flux and its variations, Space Sci. Rev., 16, 771-802, 1974.

Solanki, S. K. and Unruh, Y. C.: A model of the wavelength dependence of solar irradiance variations, Astron. Astrophys., 329, 747-753, 1998.

Talkner, P. and Weber, R. O.: Power spectrum and detrended fluctuation analysis: application to daily temperatures, Phys. Rev. E, $62,150-160,2000$.
Tobiska, W. K., Woods, T., Eparvier, F., Viereck, R., Floyd, L., Bouwer, D., Rottman, G., and White, O. R.: The SOLAR2000 empirical solar irradiance model and forecast tool, J. Atmos. Sol.-Terr. Phys., 62, 1233-1250, 2000.

Tzanis, C., Tsivola, E., Efstathiou, M., and Varotsos, C.: Forest fires pollution impact on the solar UV irradiance at the ground, Fresen. Environ. Bull., 18, 2151-2158, 2009.

Varotsos, C.: Power-law correlations in column ozone over Antarctica, Int. J. Remote Sens., 26, 3333-3342, 2005.

Varotsos, C., Alexandris, D., Chronopoulos, G., and Tzanis, C.: Aircraft observations of the solar ultraviolet irradiance throughout the troposphere, J. Geophys. Res., 106, 14843-14854, 2001.

Varotsos, C. A., Milinevsky, G., Grytsai, A., Efstathiou, M., and Tzanis, C.: Scaling effect in planetary waves over Antarctica, Int. J. Remote Sens., 29, 2697-2704, 2008.

Varotsos, C., Efstathiou, M., Tzanis, C., and Deligiorgi, D.: On the limits of the air pollution predictability: the case of the surface ozone at Athens, Greece, Environ. Sci. Pollut. R., 19, 295-300, 2012.

Varotsos, C. A., Melnikova, I., Efstathiou, M. N., and Tzanis, C.: $1 / f$ noise in the UV solar spectral irradiance, Theor. Appl. Climatol., 111, 641-648, 2013a.

Varotsos, C. A., Melnikova, I., Efstathiou, M. N., and Tzanis, C.: On the $1 / f$ noise in the UV solar spectral irradiance, Theor. Appl. Climatol., 114, 725-727, 2013b.

Varotsos, P. A., Sarlis, N. V., and Skordas, E. S.: Detrended fluctuation analysis of the magnetic and electric field variations that precede rupture, Chaos, 19, 023114, doi:10.1063/1.3130931, 2009.

Varotsos, P. A., Sarlis, N. V. and Skordas, E. S.: Natural Time Analysis: The New View of Time. Precursory Seismic Electric Signals, Earthquakes and other Complex Time Series, SpringerVerlag, Berlin/Heidelberg, Germany, 2011.

Weber, R. O. and Talkner, P.: Spectra and correlations of climate data from days to decades, J. Geophys. Res., 106, 20131-20144, 2001.

Xue, Y., Ai, J. W., Wan, W., Guo, H. D., Li, Y. J., Wang, Y., Guang, J., Mei, L. L., and Xu, H.: Grid-enabled highperformance quantitative aerosol retrieval from remotely sensed data, Comput. Geosci.-UK, 37, 202-206, 2011.

Ziemke, J. R., Chandra, S., Herman, J., and Varotsos, C.: Erythemal weighted ultraviolet trends over northern latitudes, Radiat. Prot. Dosim., 91, 157-160, 2000. 